\title{
MedienPädagogik
}

Zeitschrift für Theorie und Praxis der Medienbildung

\section{Die Bedeutung der Usability für das Lernen mit digitalen Medien}

Marios Karapanos, Cathrin Becker und Eva Christophel

\begin{abstract}
Zusammenfassung
Im Rahmen einer quantitativen Feldstudie wurde der Zusammenhang zwischen der System Usability eines digitalen Lernmediums und dem erzielten Lernerfolg in einem computerbasierten Unterrichtssetting untersucht. 31 Schülerinnen und Schüler zweier gymnasialer Biologie-Leistungskurse absolvierten dazu eine webbasierte Lerneinheit zu einem Thema der Zellbiologie. Es zeigte sich eine mittlere Korrelation zwischen der Usabilitybewertung und dem Ergebnis eines Leistungstests $(r(28)=.384, p<.05)$. Der Zusammenhang erwies sich auch unter Kontrolle lernerfolgsrelevanter Personenmerkmale mittels multipler Regression als stabil. Gemeinsam erklären Vorwissen, numerische Verarbeitungskapazität, Aktualmotivation und System Usability 52.9\% der beobachteten Leistungstestvarianz. Ferner zeigte sich ebenso eine mittlere Korrelation zwischen System Usability und dem Interesse der Schüler an der Lernaufgabe $(r(28)=.396, p<.05)$. Usability erscheint damit als bedeutsames Interaktionsmerkmal für das Lernen mit digitalen Medien. In der durchgeführten Studie erklärte sie substantiell interindividuelle Unterschiede bei der computergestützten Wissensaneignung und stellt damit möglicherweise eine wesentliche Gelingensbedingung für die Digitalisierung des Lehrens und Lernens dar.
\end{abstract}

The Importance of Usability for Learning with Digital Media.

\begin{abstract}
In a field study we examined the relationship between the system usability of a digital learning medium and the learning success of computer-assisted instruction quantitatively. 31 students from two advanced biology courses at a German secondary school took a webbased lesson on cell biology. We found a medium correlation between subjective usability ratings and test scores $(r(28)=.384, p<.05)$. The relationship remained stable even after controlling for personality traits and states relevant to learning success by using multiple regression. Prior knowledge, numerical reasoning, current motivation, and system usability accounted for $52.9 \%$ of the observed test score variance. Furthermore, we found a medium correlation between system usability and students interest in the learning task $(r(28)=.396$, $p<.05)$. We therefore conclude that usability seems to be a significant attribute of interaction when students learn with digital media. The results of our study revealed that usability explained substantial individual differences in computer assisted knowledge acquisition and appears to be an essential success factor for the digital transformation of teaching and learning.
\end{abstract}




\section{Einleitung}

Digitale Medien entwickeln sich zu einem zunehmend etablierten Instrument des Lehrens und Lernens in schulischen und hochschulischen Bildungskontexten. Nach Ergebnissen des Länderindikators 2016 nutzt etwa die Hälfte der Lehrpersonen der Sekundarstufe I an deutschen Schulen bereits mindestens einmal pro Woche Computer im Unterricht, während lediglich 5.2 Prozent der Lehrenden keinen Computer einsetzen (Lorenz, Endberg, und Eickelmann 2016). International ist der Trend zur Computernutzung noch deutlicher ausgeprägt (Eickelmann et al. 2014). Untersuchungen an deutschen Hochschulen zeigen jedoch, dass die Integration digitaler Medien in den Lern- und Lehralltag weit langsamer und weniger durchgreifend verläuft, als erwartet (Fischer 2013; Persike und Friedrich 2016). Gemessen am Potential digitaler Lernmedien attestieren komparative Evaluationsstudien zur Lernwirksamkeit digitaler Lehre überwiegend nur geringe Vorteile gegenüber konventionellen Lehrmethoden (Means et al. 2010; Mo et al. 2015; Waxman, Lin, und Michko 2003). Die Usability der eingesetzten digitalen Lernmedien, also deren Eignung zur kontextbezogenen effektiven, effizienten und zufriedenstellenden Nutzung, ist in diesem Zusammenhang wenig untersucht (Karapanos 2015). Obschon der wesentliche Einfluss einer einfachen Nutzbarkeit auf den Adoptionsprozess von Technologien bekannt (Venkatesh et al. 2003) und Usability als Qualitätsattribut interaktiver Systeme umfassend akzeptiert ist (Nielsen 1994), werden die sich daraus ergebenden Implikationen für die Bildungsforschung und -praxis bisher kaum wahrgenommen und auch in der Medienpädagogik nicht ausreichend rezipiert.

Studien zur Exploration qualitätsdeterminierender Attribute von computergestütztem Lernen weisen auf die herausragende Bedeutung einer einfachen Nutzbarkeit digitaler Lernmedien hin. Dies gilt sowohl für die Akzeptanz durch die Lernenden (Šumak, Heričko, und Pušnik 2011) als auch für die allgemeine Qualitätsbeurteilung (Jeong und Yeo 2013; Jung 2011; Paechter, Maier, und Macher 2010). Zwar existiert eine Reihe von Publikationen an der Schnittstelle von computergestütztem Lernen und Usability. Zumeist beziehen sich diese aber auf heuristikgeleitete Gestaltungsempfehlungen und Bewertungsansätze (Mehlenbacher et al. 2005; Reeves et al. 2002; Squires und Preece 1999). Die für die Medienpädagogik grundlegende Frage, inwiefern die Usability eines Lernmediums direkt die Aufnahme und Verarbeitung von Informationen und damit den Lernprozess beeinflusst, ist bisher kaum untersucht. Gerade für die Mediendidaktik, die sich als Teilgebiet der Medienpädagogik u.a. mit dem Einsatz von Unterrichtsmedien und deren Kopplung an Unterrichtsinhalte und -ziele befasst, ist die Frage der durch das Medium angeregten Informationsverarbeitungsprozesse aber entscheidend.

Der Zusammenhang der Usability eines Lernmediums und der Aufnahme und Verarbeitung von Informationen ergibt sich aus theoretischer Perspektive aus den Implikationen der Cognitive Load Theory, was im zweiten Kapitel näher dargestellt 
wird. Die hier dargestellte Studie fusst auf einer quantitativ ausgerichteten und psychologisch orientierten Forschungslogik und orientiert sich an den damit verbundenen Begriffen und Konzepten.

\section{Theoretischer Hintergrund}

\section{Cognitive Load Theory}

Die Cognitive Load Theory basiert auf der weithin akzeptierten Annahme eines limitierten menschlichen Arbeitsgedächtnisses (Merriënboer und Sweller 2005; Sweller 1994; Sweller und Chandler 1994). Lernprozesse beanspruchen diese limitierte Ressource und führen somit zu einer kognitiven Belastung. Drei Belastungsarten werden unterschieden. Die intrinsische kognitive Belastung ergibt sich aus Komplexität und Schwierigkeit des Lernmaterials. Sie ist durch den zu lernenden Gegenstand und das domänenspezifische Vorwissen des Lernenden festgelegt (Sweller, Merrienboer, und Paas 1998). Die extrinsische und lernbezogene kognitive Belastung resultieren aus der Art und Weise der Darbietung des zu lernenden Inhaltes, d.h. der Darstellung und Gestaltung des Lernmaterials, bzw. durch die für den Prozess der Wissenskonstruktion notwendigen Ressourcen. Diese unterschiedlichen kognitiven Belastungen können als additive Teile einer Gesamtbeanspruchung angesehen werden. Komplexes Lernmaterial, geringes Vorwissen oder eine inadäquate Gestaltung können zu einer kognitiven Überlastung beim Lernen führen. Die Reduktion unnötiger extrinsischer kognitiver Belastung wird für die Ermöglichung und Erleichterung von Lernprozessen daher als zielführend angesehen (Brünken und Seufert 2006).

\section{Usability}

Usability (deutsch: Gebrauchstauglichkeit) ist definiert als das Ausmass der effektiven, effizienten und zufriedenstellenden Nutzung eines Produkts oder Systems durch bestimmte Nutzer in einem bestimmten Kontext zur Erreichung bestimmter Ziele (DIN EN ISO 9241-11 2016). Usability resultiert erst aus der Interaktion von Mensch und System und stellt damit keine Systemeigenschaft im engeren Sinne, sondern vielmehr ein Interaktionsmerkmal dar (Brooke 1996). Aus der Anforderung an eine effektive, effiziente und zufriedenstellende Nutzung lassen sich Gestaltungsgrundsätze für interaktive Systeme ableiten. Hierzu zählen Merkmale wie Aufgabenangemessenheit, Selbstbeschreibungsfähigkeit, Steuerbarkeit, Erwartungskonformität, Fehlerrobustheit oder Individualisierbarkeit (DIN EN ISO 9241-110 2008). Bei der Anwendung des Usability-Konzepts auf Lernmedien kann zwischen systembezogener (System) Usability und inhaltsbezogener (Content) Usability unterschieden werden 
(Nikmehr und Doroodchi 2008). Während System Usability die bereits genannten Merkmale wie Aufgabenangemessenheit und Fehlerrobustheit umfasst, fokussiert inhaltsbezogene Usability primär auf Merkmale der didaktisch angemessenen Aufbereitung der zu erlernenden Inhalte, deren technisch adäquate Umsetzung und deren Passung zu Lernstilen und Präferenzen der Nutzer. Auch die spezifische Befähigung der Lehrenden zum Einsatz digitaler Medien in der Lehre, sog. Instructor Capabilities, und die Interaktivität der Lernmedien selbst können der Content Usability zugerechnet werden (Nikmehr und Doroodchi 2008). Eine strenge Trennung beider Konzepte erscheint allerdings schwierig. So hat die Interaktivität eines Lernmediums einen klaren Lernbezug und fusst auf didaktischen Überlegungen, kann aber beispielsweise nicht losgelöst von Aspekten der Interfacegestaltung betrachtet werden. Insofern sollten die Kategorien nicht als einander ausschliessend, sondern als Merkmalsmengen mit vorhandenen Überschneidungen verstanden werden.

Der Cognitive Load Theory folgend kann angenommen werden, dass eine unzureichende Usability zu einer Erhöhung der extrinsischen kognitiven Belastung beim Lernen mit digitalen Medien führt und somit Lernen verhindert oder erschwert. Obwohl davon auszugehen ist, dass die Usability von digitalen Lernmedien von Bedeutung für den Lernprozess ist (Jeffels 2011; Niegemann et al. 2008), existieren nur wenige empirische Befunde hierzu. Tselios, Avouris, Dimitracopoulou und Daskalaki (2001) verglichen die Testleistungen von zwei Studierendengruppen, die mit inhaltlich identischen Kursen auf zwei verschiedenen Lernplattformen gelernt hatten, und massen die System Usability der beiden Plattformen mittels Fragebogen. Im Ergebnis erzielte die Gruppe signifikant bessere Testleistungen, die auch mit der in puncto System Usability signifikant besser bewerteten Lernplattform lernte. Aus den berichteten statistischen Daten lässt sich das Effektmass Cohens $d$ (Cohen 1988) bestimmen. Ein mittlerer Unterschied bei der Usabilitybewertung $(d=.55)$ führte demnach zu einem mittleren Effekt bei der Testleistung $(d=.65)$. Meiselwitz und Sadera (2008) befragten Studierende eines Blended-Learning-Kurses, der digitale und konventionelle Lernangebote enthielt, zu ihrer subjektiven Wahrnehmung von System Usability und Lernerfolg und berichten eine hohe bivariate Korrelation $(r=.83)$. Beide Studien weisen allerdings methodische Defizite auf. So fehlen beispielsweise bei (Tselios et al. 2001) Angaben zu Reliabilität und Validität der eingesetzten Messinstrumente. Die Höhe des berichteten Zusammenhangs bei (Meiselwitz und Sadera 2008) lässt ebenso Zweifel an der Validität der eingesetzten Messmethodik aufkommen. Da sowohl Usability als auch Lernerfolg über Ratingskalen gemessen wurden, liegt der Verdacht nah, dass der Zusammenhang zumindest teilweise methodenbasiert und/oder möglicherweise durch einen Halo-Effekt bedingt ist. Demnach könnte die Bewertung des Lernmediums die Bewertung des eigenen Lernfortschritts «überstrahlen» oder umgekehrt. Eine methodisch robustere Untersuchung des Zusammenhangs von System Usability und Lernerfolg erscheint damit notwendig. 


\section{Prädiktoren für den Lernerfolg}

Ob ein Lernprozess erfolgreich verläuft und in Wissenszuwachs und Kompetenzreifung beim Lerner mündet, ist von einer Reihe individueller Merkmale abhängig. Vorwissen und Intelligenz gelten als die bedeutsamsten Konstrukte bei der Erklärung interindividueller Lernerfolgsunterschiede (Stern 2015). Vorwissen kann sich dabei im Sinne domänenspezifischen Wissens direkt auf den Gegenstandsbereich des zu erlernenden Sachverhalts beziehen, es kann aber auch z.B. als metakognitives Wissen das Problemlöse- und Lernverhalten begünstigen (Gruber und Stamouli 2015). Untersuchungen zum Lernen mit hypermedialen Lernumgebungen zeigen eine positive Assoziation domänenspezifischen Vorwissens mit der Anwendung metakognitiver Lernstrategien (Moos und Azevedo 2008; Taub et al. 2014). Intelligenz gilt als guter Prädiktor für Schul- (Deary et al. 2007) und Berufserfolg (Hülsheger und Maier 2008; Kramer 2009). Für mathematische Leistung ist der Zusammenhang besonders ausgeprägt. Deary et al. (2007) berichten in einer Studie mit über 70000 britischen Schulkindern für die Mathematikschulnote eine Varianzaufklärung von 58.6\%, (Kriegbaum, Jansen, und Spinath 2015) für die bei PISA gemessene Mathematikkompetenz von $31.0 \%$. Andere Personenmerkmale weisen deutlich geringere Zusammenhänge auf. Isoliert betrachtet, zeigen sich auch für weitere Konstrukte wie domänenspezifisches Interesse (Schiefele, Krapp, und Schreyer 1993) und Motivation (León, Núñez, und Liew 2015; Steinmayr und Spinath 2009) kleine bis mittlere Effekte auf den mathematischen bzw. allgemeinen Schulerfolg. Ihre inkrementelle Erklärungskraft $\left(\Delta \mathrm{R}^{2}\right)$ über Intelligenz hinausgehend ist allerdings gering, wenn Intelligenz kontrolliert wird (Gagné und St Père 2002; Kriegbaum, Jansen, und Spinath 2015; Laidra, Pullmann, und Allik 2007).

\section{Hypothesen}

Zwar liegen Studien vor, die die Annahme empirisch stützen, die System Usability eines digitalen Lernmediums könne eine wesentliche Einflussgrösse für den Lernprozess darstellen. Jedoch erschien eine Präzisierung und Überprüfung der berichteten Befunde in der Unterrichtspraxis erforderlich. Auf Grund der hohen Relevanz von Personenmerkmalen für den Lernprozess sollten diese im Untersuchungsdesign angemessenen einbezogen werden, um sie in der statistischen Analyse als Einflussgrössen berücksichtigen zu können. Überdies erschien die Frage relevant, zu klären, ob die System Usability auch das Interesse am Lernmedium beeinflussen kann, ob also gebrauchstauglichere Lernmedien tendenziell auch als interessanter wahrgenommen werden. Interesse soll dabei also nicht im Sinne eines domänenspezfischen Merkmals als Prädiktor in die Untersuchung aufgenommen, sondern als aufgabenspezifisches Kriterium betrachtet werden. 
Aufbauend auf den Implikationen der Cognitive Load Theory sowie den bereits angeführten empirischen Ergebnissen wurden in der vorliegenden Studie folgende Hypothesen überprüft:

H1) Zwischen System Usability und Interesse am Lernmedium besteht ein positiver Zusammenhang.

H2) Zwischen System Usability und Lernerfolg besteht ein positiver Zusammenhang.

Da auf Basis vorangegangener Studien davon auszugehen war, dass die Merkmale Vorwissen, Intelligenz und Motivation den Lernprozess begünstigen könnten, wurden diese im Untersuchungsdesign als mögliche Kovariablen berücksichtigt.

\section{Methode}

\section{Untersuchungsrahmen}

Die Erhebungen wurden Ende 2015 und Anfang 2016 an einem Gymnasium in Landau (Rheinland-Pfalz) im Rahmen des Projekts OpenMINTlabs durchgeführt. Ziele des Projekts sind Entwicklung und Einsatz von Blended-Learning-Lab-Konzepten, sogenannten virtuellen Laboren, die Online- und Präsenzangebote vereinen und in den ingenieur- und naturwissenschaftlich/technischen Grundlagenfächern der Verbundpartner angeboten werden. Dabei werden ergänzend zu realen Grundlagenlaboren in den MINT-Studiengängen der beteiligten Hochschulen virtuelle Laborversuche entwickelt und in die Hochschullehre integriert. Neben dem Einsatz in der Hochschullehre ist ein Ziel des Projekts, die virtuellen Labore interessierten regionalen Partnerschulen zur Verfügung zu stellen. Für den Einsatz im Schulunterricht werden die virtuellen Labore didaktisch überarbeitet und dem Niveau der gymnasialen Oberstufe angepasst. Einsatzgebiete in Schulen sind vor allem die Fächer Physik, Biologie und Chemie. Die virtuellen Labore bieten Lehrkräften der MINT-Fächer die Möglichkeit, ihren herkömmlichen Präsenzunterricht zu erweitern. Die Schüler können verschiedene virtuelle Versuche innerhalb des Unterrichts selbstständig durchführen, ohne direkt mit gefährlichen Stoffen oder nicht in ausreichender Stückzahl vorhandenen Geräten konfrontiert zu werden. 


\section{Messinstrumente}

System Usability

Für die quantitative Bestimmung der System Usability von interaktiven Systemen haben sich standardisierte Fragebogeninstrumente als zielführend erwiesen. In der vorliegenden Untersuchung kam die System Usability Scale (SUS) von Brooke (1996, 2013) zum Einsatz. Die Skala verhält sich robust gegenüber verschiedenen Interfaceklassen, wird als valide bewertet (Bangor, Kortum, und Miller 2008) und führt schon bei kleinen Stichproben zu konsistenten Ergebnissen (Tullis und Stetson 2004). Konzipiert als Likertskala, besteht die SUS aus 10 Items («Ich kann mir sehr gut vorstellen, das System regelmässig zu nutzen.»), die über 5-stufige bipolare Ratingskalen beantwortet werden. Fünf positiv gepolte Items alternieren mit fünf negativ gepolten Items. Nach Angleichung der Polarität wird eine Summenskala errechnet und eine Skalierung auf den Wertebereich von 0 bis 100 vorgenommen, wobei 100 für den bestmöglichen Wert steht. Neben einfacher Nutzbarkeit und einer guten Testökonomie sprechen vor allem die weite Verbreitung und das damit verbundene, einfach zu realisierende Benchmarking für den Einsatz der SUS. Werte oberhalb von 85 gelten als exzellent, bis 73 als gut und Werte kleiner 50 werden als inakzeptabel interpretiert (Bangor, Kortum, und Miller 2008). In vorangegangenen Untersuchungen hat sich die SUS auch für die Messung der System Usability von digitalen Lernmedien als adäquat und fruchtbar erwiesen (Harrati et al. 2016; Karapanos 2015; Orfanou, Tselios, und Katsanos 2015). Eine umfassende Betrachtung der SUS findet sich bei Sauro (2011).

\section{Intelligenz}

Als Mass für die kognitive Leistungsfähigkeit wurde die Verarbeitungskapazität der Schüler mittels vier Aufgabenbereichen aus dem Berliner Intelligenzstruktur-Test (BIS 4; Jäger, Süß, und Beauducel 1997), der die Intelligenzleistung hinsichtlich verschiedener Aspekte klassifiziert, erfasst. Um bei der Erfassung den Erfordernissen der in der Untersuchung eingesetzten virtuellen Labore Rechnung zu tragen, wurden Aufgaben ausgewählt, die Inhalte und Operationen berücksichtigen, die den Schülern die Bearbeitung der Labore erleichtern. Demgemäss wurden die Dimensionen zahlen- und sprachgebundenes Denken erhoben. Die Bearbeitung wurde durch den Versuchsleiter unter Zeitvorgaben angeleitet nach den Instruktionsvorgaben des BIS 4. Die nummerische Verarbeitungskapazität wurde mittels rechnerischem Denken und Tabellenlesen erfasst. Der verbale Einfallsreichtum wurde in zwei Bereichen erfasst. Zum einen sollte für einen verbal vorgegebenen sozialen Sachverhalt möglichst viele verschiedene Erklärungsmöglichkeiten angegeben werden und zum anderen sollte aus drei vorgegebenen Substantiven möglichst viele Sätze mit möglichst verschiedenem Inhalt gebildet werden. 


\section{Aktualmotivation}

Die aktuelle Motivation der Schüler wurde mit dem Fragebogen zur aktuellen Motivation (FAM) von Rheinberg, Vollmeyer und Burns (2001) in drei Dimensionen gemessen (13 Items, siebenstufige bipolare Ratingskala). Erfasst wurden die empfundene Herausforderung («Ich bin sehr gespannt, wie gut ich hier abschneiden werde.»), die Erfolgswahrscheinlichkeit («Ich glaube, das kann jeder schaffen.») und die Misserfolgsbefürchtung ( «Es ist mir etwas peinlich, hier zu versagen.»). Die vierte Dimension Interesse wurde nach der Bearbeitung des virtuellen Labors mit fünf an die Lernsituation angepassten Items des FAM erfasst («Nach dem Lesen der «Orientierung» erscheint mir die Aufgabe sehr interessant»). Alle vier Dimensionen des FAM wurden jeweils zu Summenskalen verrechnet.

\section{Vorwissen}

Zur Kontrolle des Vorwissens wurden die Schüler nach ihrer letzten Zeugnisnote (deutsche Schulnotenskala von 1: sehr gut bis 6: ungenügend) im Fach Biologie gefragt sowie danach, ob sie das Thema des virtuellen Labors bereits im Unterricht behandelt hatten oder nicht (dichotomes Merkmal: ja/nein).

\section{Lernerfolg}

Die Messung des Lernerfolgs erfolgte mittels eines speziell auf die Lernziele des virtuellen Labors hin entwickelten Multiple-Choice-Tests. Der Test umfasste 10 Items mit jeweils 3 bis 5 Antwortoptionen («Was sind Bestandteile eines idealen Mediums für eine Zellkultur mit menschlichen Zellen? a) Lichtquelle; b) Endotoxine; c) Wachstumsfaktoren»), von denen mindestens eine aber auch mehrere Antworten korrekt sein konnten. Insgesamt mussten die Schüler so über 42 Antwortoptionen entscheiden. Die Testleistung wurde als Summe der korrekt gegebenen Antworten gebildet.

\section{Lernmaterialien und Lernumgebung}

Virtuelle Labore bieten die Möglichkeit einer systematischen, didaktisch strukturierten Erfahrungsgewinnung, die sowohl motivationale als auch kognitive Anreize bereitstellt. Die im Rahmen des Projekts entwickelten virtuellen Labore bestehen aus fünf modularen Kapiteln: 1. der «Orientierung», 2. den theoretischen «Grundlagen», 3. dem «Experiment», das sowohl einen virtuellen Versuch als auch ein reales Laborexperimente enthalten kann, im konkreten Fall jedoch auf einen virtuellen Versuch beschränkt war, 4. der «Anwendung» mit Aufgaben und Beispielen aus der Berufspraxis (aus Zeitgründen nicht im Schülerlabor enthalten) und 5. der abschliessenden «Reflexion» mit Ergebnissicherung. Das bei der vorliegenden Studie eingesetzte virtuelle Labor «Zellkultur» beschäftigt sich unter anderem mit dem typischen Verlauf eines Zellkulturwachstums anhand einer Wachstumskurve, mit drei Parametern 
für die Zellkultur und mit der Analyse des Wachstums einer Zellkultur anhand von Wachstumskurven. Die Durchführung des virtuellen Labors dauert ca. 45 Minuten.

Das Kapitel «Orientierung» des virtuellen Labors «Zellkultur» dient der motivierenden Einführung in das Thema. Im Kapitel «Grundlagen» werden die für das Verständnis notwendigen theoretischen Aspekte zu Zellkulturen erläutert. Anhand von interaktiven Grafiken können die Grundlagen zu den verschiedenen Phasen eines Zellkulturwachstums und zu Bestandteilen des Zellkulturmediums erarbeitet werden. Im Kapitel «Experiment», das in Abb. 1 dargestellt ist, soll mit Hilfe einer Simulation ein Medium für die Kultur der Zellen zusammengestellt werden, das einen für das Wachstum der Zellen geeigneten pH-Wert besitzt. Das Kapitel «Reflexion» schliesst die Lerneinheit ab. Die Lerner werden zur Überprüfung des Lernerfolgs angeregt und die gewonnenen Erkenntnisse zusammengefasst.

\section{Erhebung}

Die Erhebung fand im Regelunterricht zweier Biologie-Leistungskurse in einem PCRaum der Schule statt. Wegen Einschränkungen bei der zur Verfügung stehenden Internetbandbreite an der Schule arbeiteten die Schüler paarweise an einem PC. Die Daten wurden mittels Papierfragebögen erfasst. Die Untersuchung dauerte inklusive Bearbeitung des virtuellen Labors und Beantwortung der Fragebögen eine Doppelstunde (90 Minuten). Um die genannten Schülermerkmale zu erfassen, wurden vor der Bearbeitung der Lerneinheiten im ersten Schritt mittels Aufgaben aus dem Berliner Intelligenzstruktur-Test (Jäger, Süß, und Beauducel 1997) numerische Verarbeitungskapazität und verbaler Einfallsreichtum der Teilnehmer erfasst. Im nächsten Schritt bearbeiteten die Schüler das virtuelle Labor selbständig. Nach einer Explorationsphase erhielten die Schüler einen Fragebogen zur Erhebung von drei Facetten der Aktualmotivation (Rheinberg, Vollmeyer, und Burns 2001). Nach einer weiteren Bearbeitungsphase wurde der Lernerfolg mittels Leistungstest gemessen. Im Anschluss erhielten die Schüler im vierten Schritt einen weiteren Fragebogen, mit dem ihr Alter, biologisches Geschlecht, ihre letzte Zeugnisnote im Fach Biologie, ihre Vertrautheit mit dem Thema, das Interesse (Rheinberg et al. 2001) und abschliessend die System Usability (Brooke 1996) des virtuellen Labors erhoben wurden. Die Schüler erhielten kein Feedback über ihr Ergebnis im Leistungstest, um anschliessende Messungen nicht zu beeinflussen. 

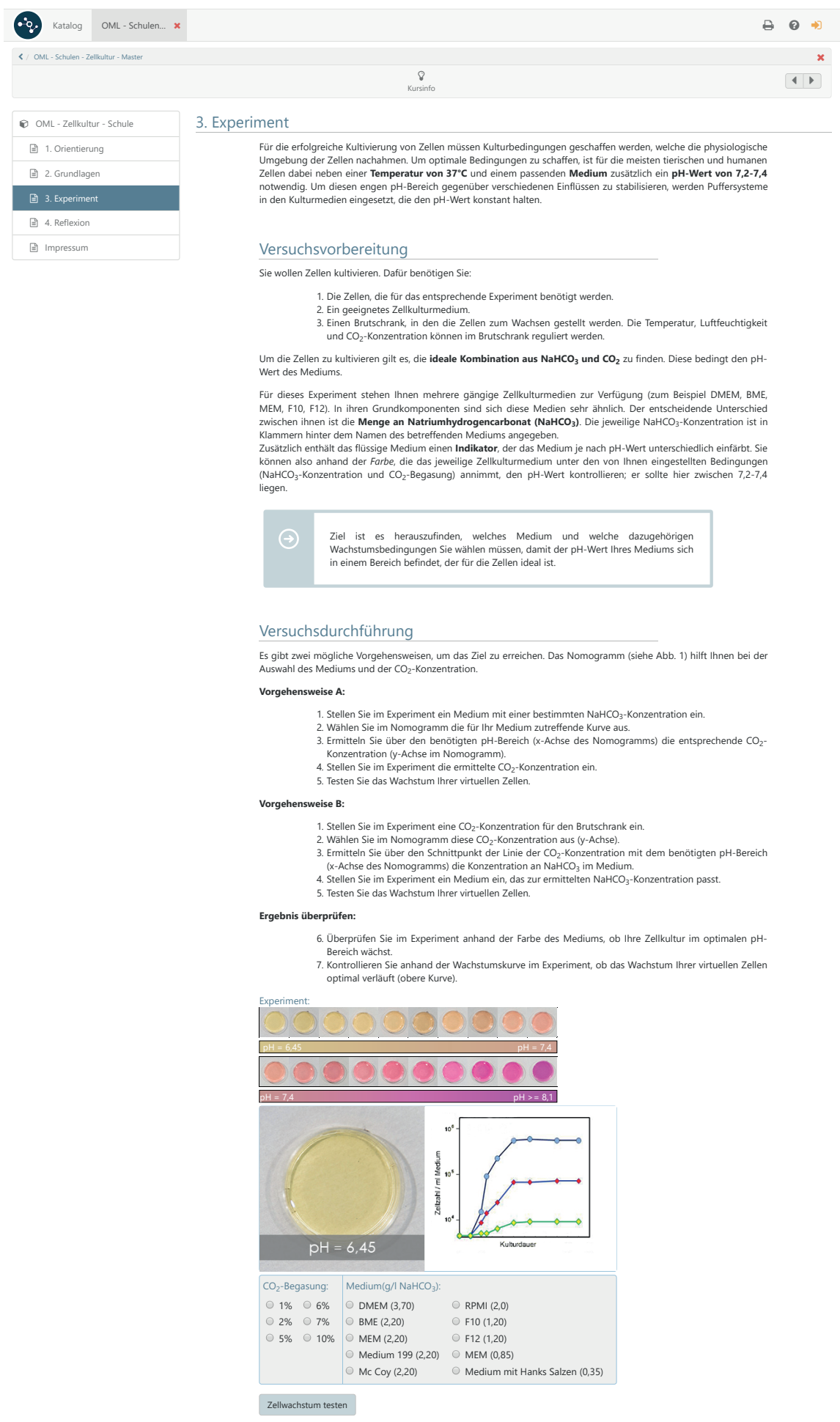

Abb. 1.: Screenshot aus dem virtuellen Labor «Zellkultur», entwickelt an der Hochschule Kaiserslautern. (https://olat.vcrp.de/auth/RepositoryEntry/1502281875/) 


\section{Stichprobenbeschreibung}

Die Stichprobe bestand aus 31 Schülern (10 männlich, ein(e) Proband(in) machte keine Angaben zum Geschlecht) aus zwei verschiedenen Biologie-Leistungskursen eines Gymnasiums. Das Alter der Schüler lag zwischen 16 und 19 Jahren $(M=16.77, S D=$ .88). Die schulische Leistung im Fach Biologie reichte von Note 1,0 bis Note 4,0 ( $M=$ 2.03, $S D=.83$ ). 18 Schüler hatten das Thema des virtuellen Labors bereits einmal im Unterricht behandelt. Für 13 von ihnen war das Thema neu.

\section{Skalen}

Alle eingesetzten Skalen wiesen eine zufriedenstellende interne Konsistenz auf (siehe Tabelle 1). Der verwendete Leistungstest zur Messung des Lernerfolgs kann ebenfalls als zufriedenstellend bewertet werden. Von 42 Antwortoptionen im Test wiesen nur 11 Schwierigkeitsindizes <.8 auf. Der Leistungstest war damit offenbar etwas zu einfach konstruiert. Da keiner der Schüler die Maximalpunktzahl erreichte, erlaubt der Test dennoch eine gute Differenzierung des erreichten Leistungsniveaus. Die System Usability des virtuellen Labors ist mit einem Mittelwert von ca. 67 als befriedigend einzustufen.

\begin{tabular}{|l|l|l|l|l|l|l|}
\hline & N & M & SD & Min & Max & $\begin{array}{l}\text { Cron- } \\
\text { bachs } \alpha\end{array}$ \\
\hline Leistungstest & 31 & 35.19 & 3.73 & 28 & 41 & .670 \\
\hline $\begin{array}{l}\text { Numerische Verarbeitungskapazität } \\
\text { (BIS) }\end{array}$ & 31 & 4.90 & 1.76 & 1 & 9 & $\star 1$ \\
\hline Verbaler Einfallsreichtum (BIS) & 31 & 8.90 & 2.65 & 3 & 13 & ${ }^{*}$ \\
\hline Interesse (FAM) & 31 & 17.26 & 5.23 & 8 & 30 & .714 \\
\hline Erfolgswahrscheinlichkeit (FAM) & 31 & 18.65 & 4.88 & 5 & 27 & .781 \\
\hline Misserfolgsbefürchtung (FAM) & 31 & 12.16 & 7.04 & 5 & 27 & .883 \\
\hline Herausforderung (FAM) & 29 & 14.34 & 4.58 & 8 & 25 & .719 \\
\hline System Usability (SUS) & 30 & 67.67 & 14.40 & 30 & 90 & .756 \\
\hline Biologienote & 31 & 2.03 & .83 & 1 & 4 & - \\
\hline
\end{tabular}

Tab. 1.: Skalenkennwerte.

SUS-Rohwerte zeigen für gewöhnlich eine rechtssteile Verteilung (Sauro 2011), so auch in dieser Untersuchung. Um für die weitere statistische Verwendung eine bessere Annäherung an die Normalverteilung zu erzielen, wurden die SUS-Rohwerte

1 Da nur zwei Aufgaben je Dimension ausgewählt wurden, ist die Berechnung eines Reliabilitätskoeffizienten an dieser Stelle nicht sinnvoll. 
invertiert, radiziert und anschliessend erneut invertiert, um die ursprüngliche Polarität nach der Transformation zu erhalten. Dies führte zu einer deutlichen Reduktion der Verteilungsschiefe. Rohwerte des Leistungstests wurden gleichsam behandelt, da auch hier eine rechtssteile Verteilung vorlag.

\section{Geschlechterunterschiede}

Bei der Differenzierung nach Geschlecht zeigten sich bei der System Usability und der Interessensubskala des FAM Unterschiede. Männliche Schüler $(M=5.11, S D=.97)$ bewerteten die Usability des virtuellen Labors besser als weibliche Schüler $(M=4.13$, $S D=1.22, t(27)=2.12, p=.043, d=.82)^{2}$. Auch schätzten sich die männlichen Teilnehmer $(M=21.00, S D=4.78)$ als interessierter am virtuellen Labor ein als die weiblichen Teilnehmer $(M=15.45, S D=4.63, t(28)=2.12, p=.032, d=.80)$. Alle übrigen gemessenen Variablen waren statistisch nicht unterscheidbar. Es sei aber an dieser Stelle auf die kleine Stichprobengrösse und die damit verbundene geringe Teststärke hingewiesen.

\section{System Usability, Lernerfolg und Interesse}

Die Untersuchung auf bivariate Zusammenhänge zeigte erwartungskonform mittlere Korrelationen ${ }^{3}$ zwischen dem Abschneiden im Leistungstest und der numerischen Verarbeitungskapazität, der System Usability und der Biologienote (siehe Tabelle 2). Zwischen den Subskalen des Fragebogens zur aktuellen Motivation (Interesse, Erfolgswahrscheinlichkeit, Misserfolgsbefürchtung, Herausforderung) zeigten sich mittlere bis hohe Zusammenhänge. System Usability und Interesse korrelierten auf mittlerem Niveau.

Beide Untersuchungshypothesen können damit als bestätigt akzeptiert werden. Um Kovariablen bei der Überprüfung des Zusammenhangs zwischen System Usability und Lernerfolg berücksichtigen zu können, wurde eine multiple Regression durchgeführt.

2 Zwar ist für die Nutzung des t-Tests die Voraussetzung homogener Varianzen verletzt, allerdings weist die grössere Gruppe die grössere Varianz auf, was nach Ramsey (1980, zitiert nach Bortz und Schuster (2010, $123 \mathrm{f.}$ )) zu einer konservativen Testentscheidung führt. Ein parallel durchgeführter Mann-Whitney-Test kam zum gleichen Ergebnis.

3 Die Bewertung von Effektmassen erfolgt gemäss der Konvention nach Cohen (1988). Demnach wird ab einer Korrelation von $r=.10$ von einem kleinen, $a b r=.30$ von einem mittleren und $a b r=.50$ von einem grossen Effekt gesprochen. 


\begin{tabular}{|l|l|l|l|l|l|l|l|l|}
\hline & 1 & 2 & 3 & 4 & 5 & 6 & 7 & 8 \\
\hline 1) Leistungstest & & & & & & & & \\
\hline $\begin{array}{l}\text { 2) Numerische Verarbei- } \\
\text { tungskapazität }\end{array}$ & $.390^{\star}$ & & & & & & & \\
\hline 3) Verbaler Einfallsreichtum & -.170 & .134 & & & & & & \\
\hline 4) System Usability & $.384^{\star}$ & .114 & -.155 & & & & & \\
\hline 5) Interesse & -.083 & .199 & -.128 & $.396^{\star}$ & & & & \\
\hline 6) Erfolgswahrscheinlichkeit & .134 & .237 & .106 & .09 & .236 & & & \\
\hline 7) Misserfolgsbefürchtung & .18 & -.018 & .192 & .054 & $.434^{\star}$ & -.171 & & \\
\hline 8) Herausforderung & -.033 & -.108 & -.165 & .158 & $.649^{\star \star}$ & .05 & $.755^{\star \star}$ & 1 \\
\hline 9) Biologienote & $-.462^{\star \star}$ & -.338 & -.059 & .125 & -.04 & $-.471^{\star \star}$ & .044 & .072 \\
\hline
\end{tabular}

Tab. 2.: Pearson-Korrelation der untersuchten Variablen. (Anmerkungen: ${ }^{\star} p<.05,{ }^{\star \star} p<.01$, $29 \leq \mathrm{n} \leq 31$ )

Aufgrund der Sensitivität von Regressionsanalysen gegenüber statistischen Ausreissern erfolgte eine Inspektion mittels Mahalanobis-Distanz, Cook'scher Distanz und Hebelwerten (Leverage-Index). Kritische Werte für Mahalanobis und Cook'sche Distanz wurden bei (Stevens 1984) entnommen. Die Beurteilung der Hebelwerte erfolgte nach einer bei (Urban und Mayerl 2011, 188) vorgeschlagenen Faustregel für kleine Stichproben. Auf Basis der Inspektion konnten keine Ausreisser innerhalb der Stichprobe identifiziert werden. Multikollinearität stellt in den vorliegenden Daten kein Problem dar. (Urban und Mayerl 2011, 232) nennen Werte für den Varianzinflationsfaktor (VIF) grösser 5 als kritisch. Alle in die Regression aufgenommenen unabhängigen Variablen weisen deutlich geringere Werte auf (siehe Tabelle 3). Die Daten eines Schülers konnte wegen Unvollständigkeit für die Berechnung der Regressionsmodelle nicht berücksichtigt werden.

Die Aufstellung des Regressionsmodells erfolgte unter Aufnahme aller Prädiktoren und anschliessender Rückwärtselimination. Dabei werden sequenziell die nichtsignifikanten Prädiktoren aus dem Modell entfernt, welche die geringste partielle Korrelation mit der Kriteriumsvariablen aufweisen. In drei Schritten wurden dabei verbaler Einfallsreichtum sowie die Motivationsfacetten Herausforderung und Erfolgswahrscheinlichkeit aus dem Modell entfernt. Die Transformation von SUS- und Leistungstestwerten führte zu einer deutlich besseren Normalverteilungsannäherung der Regressionsresiduen gegenüber den untransformierten Werten. Zusätzlich wurden alle Prädiktorvariablen zentriert.

Das resultierende Modell zeigt einen statistischen Zusammenhang zwischen dem Ergebnis im Leistungstest und den Prädiktoren System Usability, Misserfolgsbefürchtung, Vertrautheit des Themas und der numerischen Verarbeitungskapazität

4 Die Korrelationskoeffizienten der Biologienote sind negativ, da die Notenskala gegenüber allen anderen Skalen eine umgekehrte Polarität besitzt. Kleine Zahlenwerte stehen für eine hohe Merkmalsausprägung. 
$(F(4,23)=6.463, p=.001)$. Das Modell erklärt $52.9 \%$ der beobachteten Leistungstestvarianz. System Usability, numerische Verarbeitungskapazität, Vertrautheit mit dem Thema und die Motivationsdimension Misserfolgsbefürchtung zeigen erwartungskonform einen positiven Zusammenhang mit dem Abschneiden im Leistungstest.

\begin{tabular}{|l|l|l|l|l|l|l|}
\hline & B & $\begin{array}{l}\text { Stan- } \\
\text { dard- } \\
\text { fehler }\end{array}$ & B & T & p & VIF \\
\hline (Konstante) & .554 & .029 & & 19.404 & .000 & \\
\hline System Usability & .042 & .014 & .441 & 2.871 & .009 & 1.152 \\
\hline Misserfolgsbefürchtung & .005 & .002 & .324 & 2.249 & .034 & 1.014 \\
\hline Vertrautheit des Themas & .107 & .038 & .458 & 2.796 & .010 & 1.311 \\
\hline Numerische Verarbeitungskapazität & .036 & .010 & .564 & 3.666 & .001 & 1.156 \\
\hline
\end{tabular}

Tab. 3.: Regressionsanalyse auf das Ergebnis des Leistungstests. (Anmerkung: $n=30$ )

Unter Einbezug lernerfolgsrelevanter Kovariablen zeigt sich im Modell ein stabiler hochsignifikanter Zusammenhang von System Usability und dem Ergebnis des Leistungstests. Die partielle Korrelation von System Usability und Testleistung unter Berücksichtigung der übrigen Modellvariablen deutet auf einen grossen Effekt hin $\left(r_{s}(23)=.508, p=.007,95 \% \mathrm{Cl}[.178, .731]\right)$. Auch unter Kontrolle von Kovariablen kann damit die Untersuchungshypothese $\mathrm{H} 2$ angenommen werden. Die geschätzte Effektstärke liegt damit sogar noch über der der bivariaten Korrelation. Aufgrund der für einen quantitativen Forschungsansatz kleinen Stichprobe von nur 31 Probanden und der Reduktion auf ein einziges Lernsetting sollte das Ergebnis aber zurückhaltend interpretiert werden. Zwar sind die Ergebnisse erwartungskonform und bestätigen frühere Befunde (Tselios et al. 2001; Meiselwitz und Sadera 2008). Sie bedürfen aber der weitergehenden Präzisierung und Überprüfung.

\section{Diskussion und Ausblick}

Aufgrund der genannten Einschränkungen können die Ergebnisse der durchgeführten Studie nur sehr vorsichtig interpretiert werden. Sie liefern dennoch erste Hinweise dazu, dass ein Zusammenhang zwischen System Usability und dem erzielten Lernerfolg innerhalb eines computergestützten Lernszenarios bestehen kann. Trotz aller Einschränkungen können sie damit als empirischer Beleg der vorgestellten Definition von Usability herangezogen werden. Folglich erwies sich der Zusammenhang von System Usability und dem erzielten Lernerfolg sowohl in der einfachen bivariaten Korrelation wie auch bei der regressionsanalytischen Kontrolle unter Einbezug lernerfolgsrelevanter Kovariablen in der durchgeführten Studie als signifikant. Demgemäss zeigte sich, dass eine als besser wahrgenommene System Usability auch zu einem höheren Lernerfolg führte. Die Annahme einer ursächlichen Beziehung 
zwischen Lernerfolg und System Usability ist theoretisch durch die Cognitive Load Theory begründbar und wird empirisch durch die in dieser Studie gefundenen Zusammenhänge unterstützt. Eine unzureichende System Usability bindet demnach kognitive Ressourcen des Lernenden, die dann für Konstruktion und Modifikation von Wissensstrukturen nicht mehr zur Verfügung steht, was zu einer Beeinträchtigung der Lernleistung führen kann. Diese Erkenntnis zeigt, dass bei der mediendidaktischen Auswahl von medialen Unterrichtsinhalten die Usability der Inhalte bei der Erreichung der Unterrichtsziele eine grosse Rolle spielt. Im Hinblick darauf könnte ein Vorhaben künftiger Studien sein, den Zusammenhang von Usability, kognitiver Belastung und Lernleistung empirisch zu bestimmen. Eine stärkere Hinwendung zu Aspekten der Usability sowohl in der Praxis des Lehrens mit digitalen Medien wie auch in der E-Learning-Forschung erscheint damit notwendig, um zum einen kognitionsadäquate digitale Lernmedien entwickeln zu können und zum anderen ein offenbar wesentliches Merkmal der Interaktion von Lernendem und Lernmedium bei der Forschung stärker zu berücksichtigen. Vor dem Hintergrund der kleinen Stichprobe der vorliegenden Studie bedarf die Stärke des gefundenen Zusammenhangs und damit die praktische und theoretische Bedeutsamkeit jedoch weiterer Exploration und Präzision. Mit Blick auf die vorliegenden Befunden kann jedoch vermutet werden, dass beim Lernen mit digitalen Medien die System Usability ein entscheidender Faktor für einen gelingenden Lernprozess darstellt.

Die durchgeführte Studie zeigte weiterhin einen mittleren Zusammenhang zwischen System Usability und dem Interesse, mit dem sich die Schüler dem virtuellen Labor zuwendeten. Die Wirkrichtung ist hierbei allerdings weniger eindeutig als beim Lernerfolg. So könnten Schüler das virtuelle Labor als interessanter erlebt haben, weil sie das Lernen damit als effektiv, effizient und zufriedenstellend empfanden. Das Interesse am virtuellen Labor könnte aber auch zu einer geringeren Sensibilität gegenüber Usabilitydefiziten geführt und damit die Wahrnehmung und Bewertung beeinflusst haben. Eine Untersuchung von Raita und Oulasvirta (2011) kam zu dem Ergebnis, dass positive bzw. negative Erwartungshaltungen Usabilitybewertungen substantiell beeinflussen. Eine positive Erwartungshaltung führt demnach zu einer Verzerrung der Bewertung zum Positiven hin. Im vorliegenden Fall könnte das Interesse am Thema oder auch am computergestützten Unterrichtsszenario zu einem ähnlichen Effekt geführt haben.

Die in der Regressionsanalyse berücksichtigten Kovariablen führten im Wesentlichen zu erwartungskonformen Ergebnissen. Auch wenn ein Grossteil des virtuellen Labors in der Bearbeitung von Sachtexten bestand, war der verbale Einfallsreichtum offenbar von untergeordneter Bedeutung. Die numerische Verarbeitungskapazität stellte hingegen eine wesentliche Einflussgrösse für das Abschneiden im Leistungstest dar. Die letzte Zeugnisnote in Biologie erwies sich als zu grober Indikator für das Vorwissen, die Vertrautheit mit dem Thema war trotz des nur dichotomen 
Messniveaus geeignet, um Vorwissen in der Untersuchung berücksichtigen zu können. Das Vorwissen stellt für die erzielte Testleitung eine positive Einflussgrösse dar. Eine höhere Misserfolgsbefürchtung war ebenfalls positiv mit dem Leistungstestergebnis assoziiert. Das erscheint auf den ersten Blick ebenfalls plausibel, da befürchteter Misserfolg in Lern- und Leistungssituationen ein starkes Motiv darstellen kann. Dafür spräche auch die hohe Korrelation mit der Motivationsdimension Herausforderung (siehe Tabelle 2). Allerdings war das Abschneiden im Leistungstest für die teilnehmenden Schüler mit keinerlei schulischen Konsequenzen verbunden und auch der Fachlehrpersonen erhielt keine Rückmeldung über die erbrachte Testleistung. Eine mögliche Erklärung könnte darin liegen, dass die Skala hier nicht nur eine momentane, sondern auch eine zeitstabile Disposition zum Lernen aus Angst vor Misserfolg erfasste. In diesem Fall wäre auch ein konsequenzenloses negatives Abschneiden im Leistungstest ein individuell zu vermeidender Zustand. Eine alternative Erklärung wäre, dass die Wahrnehmung der Unterrichtseinheit durch die Schülerinnen und Schüler sich nicht von regulärem Unterricht unterschied, da Lernumgebung (Schule, Klassensituation) und -inhalt regulären Unterrichtsveranstaltungen entsprachen. Eine dritte Erklärung könnte in der Bearbeitung des virtuellen Labors in Zweiergruppen liegen, was eine Form externer sozialer Kontrolle des Lernprozesses darstellt.

Für die Bewertung der System Usability des virtuellen Labors wurden SUS-Werte von 30 bis 90 gemessen. 13 Schülerinnen und Schüler bewerteten die System Usability des virtuellen Labors als gut oder exzellent (SUS > 73), 4 bewerteten sie als inakzeptabel (SUS < 52). Die grosse Spannweite kann als Beleg dafür angesehen werden, dass selbst in relativ homogenen Gruppen wie zwei Biologie-Leistungskursen der gleichen Schule, ein digitales Lernmedium nicht für alle Schülerinnen und Schüler gleichermassen geeignet ist. Hieraus ergeben sich weitreichende Implikationen für die Auswahl und den Einsatz von digitalen Lernmedien.

Als offenes Forschungspotential ergibt sich die Frage nach Persönlichkeitsmerkmalen und Dispositionen, die die Usabilitywahrnehmung digitaler Lernmedien bestimmen. Naheliegend sind hier Konzepte wie Medienkompetenz oder Selbstwirksamkeitsüberzeugungen zum kompetenten Umgang mit digitalen Medien. Personen, die geübt im Umgang mit Informations- und Kommunikationstechnologien sind und Erfahrung mit verschiedenen Soft- und Hardwaresystemen besitzen, sollten auch leichter in der Lage sein, Interaktionsprinzipien wiederzuerkennen, Analogien herzustellen und so auch digitale Lernmedien effektiver und effizienter für sich nutzbar zu machen. Eine völlig ungeübte Person könnte einen Grossteil der zur Verfügung stehenden Lernzeit dafür benötigen, die Bedienung des Mediums zu erlernen (Jeffels 2011) oder mehr kognitive Ressourcen für die Orientierung bspw. in Hypertextumgebungen benötigen. Ein versierter Nutzer wäre hingegen in der Lage, unmittelbar mit der inhaltlichen Auseinandersetzung zu beginnen und könnte bei Interaktionen auch 
mit unbekannten Systemen intuitiv handeln. Stützen kann sich diese Vermutung auf Befunde aus der Technologieakzeptanzforschung. Ein in dieser Forschungslinie als wahrgenommener Bedienkomfort (Perceived Ease of Use) bezeichnetes Konstrukt wird beeinflusst von den Faktoren computerbezogene Selbstwirksamkeit, computerbezogene Angst und der Neigung zur spielerischen Computernutzung (Brown 2002; Venkatesh 2000; Venkatesh und Bala 2008). Geringere computerspezifische Selbstwirksamkeitsüberzeugungen und Technikaffinität sind für Mädchen gegenüber Jungen in der Literatur belegt (Wolffram und Winker 2005; Karapanos und Fendler 2015). Insofern könnte hier auch eine Erklärung für die gefundenen Geschlechterunterschiede hinsichtlich Interesse und Usability liegen. Auch wenn sich in der vorliegenden Untersuchung keine signifikanten korrelativen Zusammenhänge zwischen System Usability und Intelligenzfacetten ergaben, könnten kognitive Merkmale wie Intelligenz für die Usabilitywahrnehmung ebenfalls eine Rolle spielen. Campbell (2011) konnte hierzu bereits zeigen, dass räumliches Vorstellungsvermögen und die Effektivität bei der Informationssuche in Hypertextumgebungen korrespondieren.

Der Zusammenhang zwischen System Usability und Lernerfolg wurde in der vorliegenden Untersuchung durch ein lineares Modell abgebildet. Es ist allerdings davon auszugehen, dass die Annahme eines linearen Zusammenhangs nicht uneingeschränkt gültig sein kann. Während ein völlig gebrauchsuntaugliches Lernmedium das Lernen unmöglich machen kann, ist es nicht plausibel davon auszugehen, dass am oberen Ende der Skala Lernen durch eine weitere Verbesserung der System Usability eines Lernmediums quasi beliebig gefördert werden kann. Vielmehr ist davon auszugehen, dass der Zusammenhang nur so lang besteht, wie die System Usability des Lernmediums auch zu einer kognitive Belastung führt. Die System Usability könnte aus dieser Perspektive betrachtet eher eine moderierende Funktion übernehmen. Auch ist die System Usability kein zeitstabiles Merkmal. Wie Sauro (2011) berichtet, treten im Nutzungsverlauf Gewöhnungseffekte ein. Nutzer, die Websites wiederholt benutzten, bewerteten deren System Usability um durchschnittlich $11 \%$ besser als Nutzer, die die gleichen Websites zum ersten Mal nutzten. Daraus lässt sich schliessen, dass die System Usability in Fällen bedeutender ist, in denen digitale Lernmedien für einen aus Sicht des Lerners singulären oder kurzzeitigen Gebrauch gestaltet werden.

Für die Praxis kann die Schlussfolgerung gezogen werden, dass eine verstärkte Inspektion von Usability-Aspekten beim Einsatz digitaler Medien in der Lehre sinnvoll und notwendig ist und bei der mediendidaktischen Auswahl von Lehrenden im Rahmen der Planung ihres Unterrichts als grundlegender Faktor einbezogen werden sollte. Hierfür steht mit der System Usability Scale ein valides und ökonomisches Messinstrument zur Verfügung, das auch für die Bewertung digitaler Lernmedien geeignet ist. 


\section{Literatur}

Bangor, Aaron, Philip Kortum, und James T. Miller. 2008. «An Empirical Evaluation of the System Usability Scale». International Journal of Human-Computer Interaction 24 (6): 574-94. https://doi.org/10.1080/10447310802205776.

Bortz, Jürgen, und Christof Schuster. 2010. Statistik für Human- und Sozialwissenschaftler. 7., vollständig überarbeitete und erweiterte Auflage. Berlin, Heidelberg: Springer-Verlag Berlin Heidelberg.

Brooke, John. 1996. «SUS - A quick and dirty usability scale». In Usability Evaluation In Industry, herausgegeben von Patrick W. Jordan, 189-94. London Bristol: Taylor \& Francis.

Brooke, John. 2013. «SUS: A Retrospective». Journal of Usability Studies 8 (2): 29-40.

Brown, Irwin T. J. 2002. «Individual and Technological Factors Affecting Perceived Ease of Use of Web-Based Learning Technologies in a Developing Country». The Electronic Journal of Information Systems in Developing Countries 9 (0). http://144.214.55.140/ojs2/index.php/ ejisdc/article/view/50.

Brünken, Roland, und Tina Seufert. 2006. «Aufmerksamkeit, Lernen, Lernstrategien.» In Handbuch Lernstrategien, herausgegeben von Heinz Mandl und Helmut Felix Friedrich, 27-37. Göttingen u.a.

Campbell, Susan G. 2011. «Users' spatial abilities affect interface usability outcomes». College Park: University of Maryland. http://drum.lib.umd.edu/handle/1903/11789.

Cohen, Jacob. 1988. Statistical power analysis for the behavioral sciences. 2. Aufl. Hillsdale, NJ [u.a.]: Erlbaum.

Deary, Ian J., Steve Strand, Pauline Smith, und Cres Fernandes. 2007. «Intelligence and Educational Achievement». Intelligence 35 (1): 13-21. https://doi.org/10.1016/j.intell.2006.02.001.

DIN EN ISO 9241-11. 2016. Ergonomie der Mensch-System-Interaktion - Teil 11: Gebrauchstauglichkeit: Begriffe und Konzepte (ISO/DIS 9241-11:2015); Deutsche und Englische Fassung prEN ISO 9241-11:2015. 11. Berlin: Beuth.

DIN EN ISO 9241-110. 2008. Ergonomie der Mensch-System-Interaktion - Teil 110: Grundsätze der Dialoggestaltung: (ISO 9241-110:2006); Deutsche Fassung EN ISO 9241-110:2006. September 2008. DIN-Normen / Deutsches Institut für Normung. - Berlin : Beuth, 1917- 110. Berlin: Beuth.

Eickelmann, Birgit, Heike Schaumburg, Kerstin Drossel, und Ramona Lorenz. 2014. «Schulische Nutzung von neuen Technologien in Deutschland im internationalen Vergleich.» In ICILS 2013. Computer- und informationsbezogene Kompetenzen von Schülerinnen und Schülern in der 8. Jahrgangsstufe im internationalen Vergleich., herausgegeben von Wilfried Bos, Birgit Eickelmann, Julia Gerick, Frank Goldhammer, Heike Schaumburg, Knut Schwippert, Martin Senkbeil, Renate Schulz-Zander, und Heike Wendt, 197-230. Münster, New York: Waxmann.

Fischer, Helge. 2013. E-Learning im Lehralltag: Analyse der Adoption von E-Learning-Innovationen in der Hochschullehre. Wiesbaden: Springer VS. 
Gagné, Françoys, und François St Père. 2002. «When IQ is controlled, does motivation still predict achievement?» Intelligence 30 (1): 71-100. https://doi.org/10.1016/S01602896(01)00068-X.

Gruber, Hans, und Eleni Stamouli. 2015. «Intelligenz und Vorwissen». In Pädagogische Psychologie, herausgegeben von Elke Wild und Jens Möller, 25-44. Springer-Lehrbuch. Springer Berlin Heidelberg. https://doi.org/10.1007/978-3-642-41291-2_2.

Harrati, Nouzha, Imed Bouchrika, Abdelkamel Tari, und Ammar Ladjailia. 2016. «Exploring user satisfaction for e-learning systems via usage-based metrics and system usability scale analysis». Computers in Human Behavior 61 (August): 463-71. https://doi.org/10.1016/j. chb.2016.03.051.

Hülsheger, Ute R., und Günter W. Maier. 2008. «Persönlichkeitseigenschaften, Intelligenz und Erfolg im Beruf: Eine Bestandsaufnahme internationaler und nationaler Forschung». Psychologische Rundschau 59 (2): 108-22. https://doi.org/10.1026/0033-3042.59.2.108.

Jäger, Adolf Otto, Heinz-Martin Süß, und Andre Beauducel. 1997. «Berliner Intelligenzstruktur - Test. Form 4». In Handbuch wirtschaftspsychologischer Testverfahren, herausgegeben von Werner Sarges und Heinrich Wottawa, 95-101. Lengerich [u.a.]: Pabst Science Publ.

Jeffels, Peter. 2011. «Usability, in relation to e-learning projects». https://www.abdn.ac.uk/ eLearning/documents/Jeffels_Usability2011.pdf.

Jeong, Hwa-Young, und Sang-Soo Yeo. 2013. «The Quality Model for E-Learning System with Multimedia Contents: A Pairwise Comparison Approach». Multimedia Tools and Applications 73 (2): 887-900. https://doi.org/10.1007/s11042-013-1445-5.

Jung, Insung. 2011. «The Dimensions of E-Learning Quality: From the Learner's Perspective». Educational Technology Research and Development 59 (4): 445-64. https://doi.org/10.1007/ s11423-010-9171-4.

Karapanos, Marios. 2015. «Gebrauchstauglichkeit und Nützlichkeit. Usability und wahrgenommener Nutzen digitaler Lernangebote.» In Wissensgemeinschaften 2015, herausgegeben von Thomas Köhler, Nina Kahnwald, und Eric Schoop, 125-34. Dresden: TUDpress. http:// www.qucosa.de/recherche/frontdoor/?tx_slubopus4frontend\%5bid\%5d=urn:nbn:de:b sz:14-qucosa-181566.

Karapanos, Marios, und Jan Fendler. 2015. «Lernbezogenes Mediennutzungsverhalten von Studierenden der Ingenieurswissenschaften. Eine geschlechterkomparative Studie.» Journal of Technical Education (JOTED) 3 (1). http://www.journal-of-technical-education.de/ index.php/joted/article/view/33.

Kramer, Jochen. 2009. «Metaanalytische Studien zu Intelligenz und Berufsleistung in Deutschland». Bonn: Rheinische Friedrich-Wilhelms-Universität Bonn. http://hss.ulb.uni-bonn. de/2009/1708/1708.htm.

Kriegbaum, Katharina, Malte Jansen, und Birgit Spinath. 2015. «Motivation: A predictor of PISA's mathematical competence beyond intelligence and prior test achievement». Learning and Individual Differences 43: 140-48. https://doi.org/10.1016/j.lindif.2015.08.026. 
Laidra, Kaia, Helle Pullmann, und Jüri Allik. 2007. «Personality and intelligence as predictors of academic achievement: A cross-sectional study from elementary to secondary school». Personality and Individual Differences 42 (3): 441-51. https://doi.org/10.1016/j. paid.2006.08.001.

León, Jaime, Juan L. Núñez, und Jeffrey Liew. 2015. «Self-determination and STEM education: Effects of autonomy, motivation, and self-regulated learning on high school math achievement». Learning and Individual Differences 43: 156-63. https://doi.org/10.1016/j. lindif.2015.08.017.

Lorenz, Ramona, Manuela Endberg, und Birgit Eickelmann. 2016. «Unterrichtliche Nutzung digitaler Medien von Lehrkräften der Sekundarstufe I in Deutschland und im Bundesländervergleich Aktuelle Ergebnisse für 2016 und der Trend seit 2015». In Schule digital - der Länderindikator 2016: Kompetenzen von Lehrpersonen der Sekundarstufe I im Umgang mit digitalen Medien im Bundesländervergleich, herausgegeben von Wilfried Bos, Ramona Lorenz, Manuela Endberg, Birgit Eickelmann, Rudolf Kammerl, und Stefan Welling, 80-109. Münster New York: Waxmann.

Means, Barbara, Yukie Toyama, Robert Murphy, Marianne Bakia, und Karla Jones. 2010. Evaluation of Evidence-Based Practices in Online Learning: A Meta-Analysis and Review of Online Learning Studies. US Department of Education. http://eric.ed.gov/?id=ED505824.

Mehlenbacher, Brad, Leslie Bennett, Tammy Bird, Melonie Ivey, Jan Lucas, Janet Morton, und Lisa Whitman. 2005. «Usable e-learning: A conceptual model for evaluation and design». In Proceedings of $\mathrm{HCl}$ International 2005: 11th International Conference on Human-Computer Interaction, 4:1-10. http://www4.ncsu.edu/unity/users/b/brad_m/web/research/elearning05.pdf.

Meiselwitz, Gabriele, und W. Sadera. 2008. «Investigating the connection between usability and learning outcomes in online learning environments». Journal of Online Learning and Teaching 4 (2): 234-42.

Merriënboer, Jeroen J. G. van, und John Sweller. 2005. «Cognitive Load Theory and Complex Learning: Recent Developments and Future Directions». Educational Psychology Review 17 (2): 147-77. https://doi.org/10.1007/s10648-005-3951-0.

Mo, Di, Weiming Huang, Yaojiang Shi, Linxiu Zhang, Matthew Boswell, und Scott Rozelle. 2015. «Computer technology in education: Evidence from a pooled study of computer assisted learning programs among rural students in China». China Economic Review 36: 131-45. https://doi.org/10.1016/j.chieco.2015.09.001.

Moos, Daniel C., und Roger Azevedo. 2008. «Self-regulated learning with hypermedia: The role of prior domain knowledge». Contemporary Educational Psychology 33 (2): 270-98. https:// doi.org/10.1016/j.cedpsych.2007.03.001.

Niegemann, Helmut M., Steffi Domagk, Silvia Hessel, Alexandra Hein, Matthias Hupfer, und Annett Zobel. 2008. Kompendium multimediales Lernen. X.media.press. Berlin: Springer.

Nielsen, Jakob. 1994. Usability Engineering. Burlington: Morgan Kaufmann. 
Nikmehr, N., und M. Doroodchi. 2008. «New paradigm in evaluating usability of E-learning system». In International Conference on Innovations in Information Technology, 2008. IIT 2008, 347-51. https://doi.org/10.1109/INNOVATIONS.2008.4781683.

Orfanou, Konstantina, Nikolaos Tselios, und Christos Katsanos. 2015. «Perceived Usability Evaluation of Learning Management Systems: Empirical Evaluation of the System Usability Scale». The International Review of Research in Open and Distributed Learning 16 (2). http:// www.irrodl.org/index.php/irrodl/article/view/1955.

Paechter, Manuela, Brigitte Maier, und Daniel Macher. 2010. «Students' expectations of, and experiences in e-learning: Their relation to learning achievements and course satisfaction». Computers \& Education 54 (1): 222-29. https://doi.org/10.1016/j.compedu.2009.08.005.

Persike, Malte, und Julius-David Friedrich. 2016. Lernen mit digitalen Medien aus der Studierendenperspektive. Sonderauswertung aus dem CHE Hochschulranking für die deutschen Hochschulen. Essen: Edition Stifterverband.

Raita, Eeva, und Antti Oulasvirta. 2011. «Too good to be bad: Favorable product expectations boost subjective usability ratings». Interacting with Computers, Cognitive Ergonomics for Situated Human-Automation Collaboration, 23 (4): 363-71. https://doi.org/10.1016/j.intcom.2011.04.002.

Reeves, Thomas C., Lisa Benson, Dean Elliott, Michael Grant, Doug Holschuh, Beaumie Kim, Hyeonjin Kim, Erick Lauber, und Sebastian Loh. 2002. "Usability and Instructional Design Heuristics for E-Learning Evaluation.» In Ed Media 2002. World Conference on Educational Multimedia, Hypermedia \& Telecommunications. Proceedings, 1615-21. http://eric. ed.gov/?id=ED477084.

Rheinberg, Falko, Regina Vollmeyer, und Bruce D. Burns. 2001. «FAM: Ein Fragebogen zur Erfassung aktuller Motivation in Lern- und Leistungssituationen». Diagnostica 47 (2): 57-66. https://doi.org/10.1026//0012-1924.47.2.57.

Sauro, Jeff. 2011. A Practical Guide to the System Usability Scale (SUS). Background, Benchmarks \& Best Practices. Denver: Measuring Usability LLC.

Schiefele, Ulrich, Andreas Krapp, und Inge Schreyer. 1993. «Metaanalyse des Zusammenhangs von Interesse und schulischer Leistung.» Zeitschrift für Entwicklungspsychologie und Pädagogische Psychologie 25 (2): 120-48.

Squires, David, und Jenny Preece. 1999. «Predicting quality in educational software: Evaluating for learning, usability and the synergy between them». Interacting with computers 11 (5): 467-483.

Steinmayr, Ricarda, und Birgit Spinath. 2009. "The importance of motivation as a predictor of school achievement». Learning and Individual Differences 19 (1): 80-90. https://doi. org/10.1016/j.lindif.2008.05.004.

Stern, Elsbeth. 2015. «Intelligence, Prior Knowledge, and Learning». In International Encyclopedia of the Social \& Behavioral Sciences (Second Edition), herausgegeben von James D. Wright, 323-28. Oxford: Elsevier. http://www.sciencedirect.com/science/article/pii/ B9780080970868920178. 
Stevens, James P. 1984. «Outliers and influential data points in regression analysis». Psychological Bulletin 95 (2): 334-44. https://doi.org/10.1037/0033-2909.95.2.334.

Šumak, Boštjan, Marjan Heričko, und Maja Pušnik. 2011. «A Meta-Analysis of e-Learning Technology Acceptance: The Role of User Types and e-Learning Technology Types». Computers in Human Behavior 27 (6): 2067-77. https://doi.org/10.1016/j.chb.2011.08.005.

Sweller, John. 1994. "Cognitive load theory, learning difficulty, and instructional design». Learning and Instruction 4 (4): 295-312. https://doi.org/10.1016/0959-4752(94)90003-5.

Sweller, John, und Paul Chandler. 1994. «Why Some Material Is Difficult to Learn». Cognition and Instruction 12 (3): 185-233. https://doi.org/10.1207/s1532690xci1203_1.

Sweller, John, Jeroen J. G. van Merrienboer, und Fred G. W. C. Paas. 1998. «Cognitive Architecture and Instructional Design». Educational Psychology Review 10 (3): 251-96. https://doi. org/10.1023/A:1022193728205.

Taub, Michelle, Roger Azevedo, François Bouchet, und Babak Khosravifar. 2014. «Can the use of cognitive and metacognitive self-regulated learning strategies be predicted by learners' levels of prior knowledge in hypermedia-learning environments?» Computers in Human Behavior 39: 356-67. https://doi.org/10.1016/j.chb.2014.07.018.

Tselios, Nikolaos K., Nikolaos M. Avouris, Angelique Dimitracopoulou, und Sophia Daskalaki. 2001. «Evaluation of Distance-Learning Environments: Impact of Usability on Student Performance». International Journal of Educational Telecommunications 7 (4): 355-79.

Tullis, Thomas S, und Jacqueline N Stetson. 2004. «A comparison of questionnaires for assessing website usability». In Usability Professional Association Conference, 1-12.

Urban, Dieter, und Jochen Mayerl. 2011. Regressionsanalyse: Theorie, Technik und Anwendung. 4., überabeitete und erweiterte Auflage. Wiesbaden: VS, Verl. für Sozialwiss.

Venkatesh, Viswanath. 2000. "Determinants of perceived ease of use: Integrating control, intrinsic motivation, and emotion into the technology acceptance model». Information systems research 11 (4): 342-365.

Venkatesh, Viswanath, und Hillol Bala. 2008. «Technology acceptance model 3 and a research agenda on interventions». Decision sciences 39 (2): 273-315.

Venkatesh, Viswanath, Michael G. Morris, Gordon B. Davis, und Fred D. Davis. 2003. «User Acceptance of Information Technology: Toward a Unified View». MIS Quarterly 27 (3): 425-78.

Waxman, Hersh C., Meng-Fen Lin, und Georgette M. Michko. 2003. «A Meta-Analysis of the Effectiveness of Teaching and Learning With Technology on Student Outcomes». Houston: University of Houston.

Wolffram, Andrea, und Gabriele Winker. 2005. «Technikhaltungen von Studienanfängerinnen und-anfängern in technischen Studiengängen. Auswertungsbericht der Erstsemesterbefragung an der TU Hamburg-Harburg im WS 03/04». TU Hamburg-Harburg. http://www.tuhh. de/agentec/publikationen/Abschlussbericht_\%20Erstsemesterbefragung_TUHH.pdf. 